\title{
e-interview
}

\section{Helen Herrman}

Helen Herrman is Professor of Public Health and Psychiatry, Australian International Health Institute, University of Melbourne, and Director of the World Health Organization Collaborating Centre for Research and Training in Mental Health. She trained at Royal Park and St Vincent's Hospitals and associated services in Melbourne. Her special interests include the promotion of mental health in poorly resourced countries and research dissemination as Secretary for Publications with the World Psychiatric Association.

\section{If you were not a psychiatrist, what} would you do?

Ever since reading the Voyage of the Beagle I have wanted to work in natural history. Then the ideas of Julian Tudor Hart and the social medicine movement in the UK sparked my interest. I'd like to read and write more in these areas and spend more time with my husband and children.

What has been the greatest impact of your profession on you personally? The people from all backgrounds I have met: colleagues in day-to-day work and faraway places, and their friends and families, as well as patients. I have lived and worked in other countries and celebrated and worshipped with people from other cultures. This must have a big impact and now I can't imagine life otherwise.

\section{What are your interests outside} of work?

Books, sports (especially those played by my sons), keeping fit (with mixed results), theatre.

Who was your most influential trainer, and why?

Dame Rosemary Rue was regional medical officer in the Oxford Regional Health Authority in the 1970s. I was a young postgraduate student and then registrar in community medicine in Oxford, and an immigrant from my native Australia. She was a model for me of a woman leader in the profession who was concerned about social justice, as well as the welfare and best training opportunities for women and men in medicine. She was president of the British Medical Association for a term, and psychiatry was for her integral to healthcare.

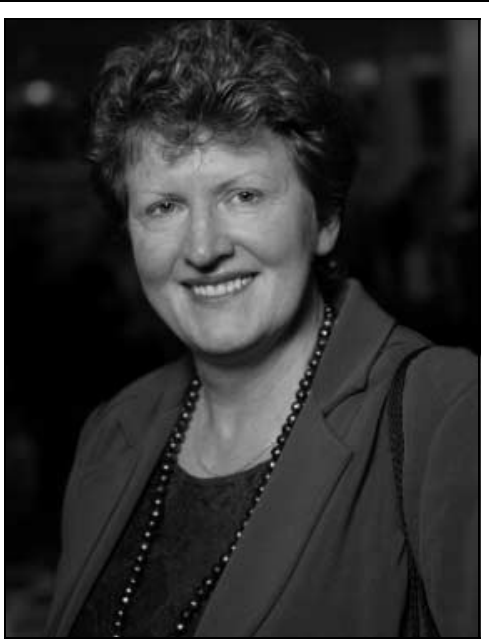

working internationally with the World Health Organization and more recently with the World Psychiatric Association.

What do you least enjoy?

Talking to people who have already made up their minds

What single change would substantially improve quality of care? Learning from consumers (or users) what matters to them, and learning from those working in poorly resourced countries how to make scarce professional resources go around.

\section{Do you think psychiatry is brainless} or mindless?

The biggest 'less' for psychiatry is its separation from mainstream public health consciousness; although this is changing with, for example, the activities of the Faculty of Public Health (of the Royal College of Physicians) in the UK and the Faculty of Public Health Medicine (of the Royal Australasian College of Physicians).

most?

George Vaillant's Adaptation to Life inspired clearer thinking about the continuities and breaks in a person's development, and associated events and circumstances. Seeing patients and considering the influence of social factors on development and mental health, and teaching about these was never the same afterwards.

What research publication has had the greatest influence on your work? Geoffrey Rose's 'Mental disorder and the strategies of prevention' (Psychological Medicine, 1993, 23, 553-555) is a model of clear thinking, and locates mental health and heart health in the same universe. Diagnoses are dimensional as well as categorical, and mental health is a population as well as an individual characteristic

What part of your work gives you the most satisfaction?

Working with groups to manage change and use the points of agreement. This was my job as director of psychiatry in a teaching hospital service in the 1990s. As part of national and state reforms in mental health services, colleagues from all disciplines and representatives of consumer and family groups worked to create a communitybased area mental health service to replace the separate institutional, general hospital and out-patient services. It was one of the hardest things to do, and not all aspects went well, but the achievements were satisfying. I have had similar experiences students into the profession?

Make it even more attractive to women, with emphasis on part-time training and the great role models in the UK.

What is the most important advice you could offer to a new trainee? Always remember the person with the illness, and talk to them as you would like your colleague to talk to your parent or sibling or child.

How would you improve clinical psychiatric training? More training for working in teams with colleagues from all disciplines, and making this practical as well as didactic, with mentoring and supervision. I would also like to see our professional training have a population focus as well as an individual one, so that psychiatrists can work as needed with decision-makers in other sectors.

What single area of psychiatric practice is most in need of development? In Australia, I suggest collaborative care with general practice or primary care, although there is a good deal of attention to this now. Research in collaborative care is needed in settings and countries of all types.

Dominic Fannon
How would you entice more medical 